Artigo Original

\title{
Tratamento multiprofissional da obesidade e sua cessação em adolescentes: efeitos no perfil hemodinâmico
}

\author{
Danilo Fernandes da Silva ${ }^{1}$ \\ Josiane Aparecida Alves Bianchini ${ }^{1}$ \\ Nelson Nardo Junior ${ }^{2}$ \\ ${ }^{1}$ Programa de Pós-graduação associado Universidade Estadual de Maringá/Universidade Estadual de \\ Londrina (UEM/UEL). Membro do Núcleo de Estudos Multiprofissional da Obesidade (NEMO), \\ Maringá, PR, Brasil \\ ${ }^{2}$ Curso de Educação Física da Universidade Estadual de Maringá, Coordenador do Núcleo de Estudos \\ Multiprofissional da Obesidade (NEMO), Maringá, PR, Brasil
}

\begin{abstract}
Resumo: Avaliar os efeitos de um Programa Multiprofissional de Tratamento da Obesidade (PMTO) e de sua cessação sobre o perfil hemodinâmico de adolescentes. Foram avaliados 19 adolescentes com excesso de peso de 10 a 18 anos. Medidas antropométricas, pressão arterial e frequência cardíaca de repouso foram incluídas na análise. Os adolescentes completaram 16 semanas do PMTO com uma equipe multiprofissional focada em promover mudanças no estilo de vida dos adolescentes. Os mesmos retornaram para avaliação após oito semanas de cessação das atividades. Após o PMTO, foi observada redução na frequência cardíaca de repouso nas meninas sem diferença significativa para o momento de cessação. Os meninos apresentaram melhora para as variáveis: circunferência abdominal, IMC, gordura relativa e massa magra após o PMTO. Após o período cessação houve aumento significativo apenas no peso. Os resultados evidenciam o impacto positivo de um PMTO demonstrando efeitos diferentes de acordo com o gênero.
\end{abstract}

Palavras-chave: Adolescente. Obesidade. Hemodinâmica.

Multiprofessional treatment of obesity and its cessation in adolescents: effects on hemodynamic profile

\begin{abstract}
To evaluate the effects of a Multiprofessional Program of Obesity Treatment (PMTO) and its cessation on hemodynamic profile in adolescents. We evaluated 19 excess of weight adolescents aged from 10 to 18 years. Anthropometric measures, resting blood pressure and resting heart rate were included in analysis. Adolescents completed 16 weeks of PMTO with a multiprofessional team focused on promote lifestyle changes in adolescents. The same adolescents returned to evaluation after eight weeks of activities cessation. After PMTO, we observed reducing in heart rate of girls without differences to cessation moment. Boys presented improvement in variables: abdominal circumference, BMI, relative body fat and lean mass after PMTO. After cessation period there was significant increase on body weight. Results evidences the positive impact of PMTO demonstrating different effects according to gender.
\end{abstract}

Keywords: Adolescent. Obesity. Hemodynamics.

\section{Introdução}

A obesidade é considerada atualmente um dos principais problemas de saúde pública enfrentados pela sociedade. A mais recente Pesquisa de Orçamentos Familiares (POF 20082009) apontou prevalência de sobrepeso de $21,7 \%$ para os meninos e $19,4 \%$ para as meninas e de obesidade de $5,9 \%$ e $4,0 \%$, em adolescentes brasileiros de 10 a 19 anos (IBGE, 2010).

Essa doença está associada a diversas complicações que podem trazer prejuízos à saúde do indivíduo. Estudos mostram que crianças e adolescentes obesos apresentam pressão arterial mais elevada em comparação com seus pares eutróficos (QUEIROZ et al., 2010; SOUZA et al., 2010), independente do gênero (POLDERMAN et al., 2011). Ademais, o aumento da pressão arterial em crianças e adolescentes pode estar relacionado com a hipertensão na idade adulta (BAO et al., 1995). Em estudo realizado com 497 adolescentes brasileiros, a prevalência de hipertensão arterial foi de $13,8 \%$ para os meninos e $11,5 \%$ para as meninas (CAMPOS et al., 2010). Ferreira e Aydos (2010) avaliaram crianças e adolescentes obesos e encontraram prevalência de $15,8 \%$ e $26,4 \%$ para o gênero masculino e feminino, respectivamente, sem diferenças significativas entre os gêneros.

Ações focadas no tratamento da obesidade têm recebido apoio de importantes órgãos de saúde do mundo, dado o aumento da doença na população ( $\underline{\mathrm{CDC}}, 2004 ; \underline{\mathrm{WHO}}, 2006)$. Em uma 
meta-análise, foi verificada associação entre a prática de exercícios aeróbios com a redução da pressão arterial sistólica e diastólica em participantes hipertensos e normotensos e em sobrepesados e eutróficos (WHELTON et al., 2002).

Programas Multiprofissionais de Tratamento da Obesidade (PMTO) para adolescentes, a partir da ação de uma equipe de profissionais da saúde, com objetivo de promover mudanças no comportamento alimentar e de atividade física são considerados importantes formas de intervenção e costumam apresentar positivos resultados em aspectos ligados à saúde de adolescentes obesos, como na pressão arterial e composição corporal (LEITE et al., 2009; CARANTI et al., 2007). Todavia, quanto aos efeitos da cessação desse tipo de programa em adolescentes sobre variáveis hemodinâmicas, até onde temos conhecimento, não há trabalhos reportados na literatura. Alguns efeitos desse período são especificamente sobre variáveis antropométricas, composição corporal e aptidão cardiorrespiratória evidenciando resultados negativos após a interrupção (ROLLAND-CACHERA et al., 2004; CARELL et al., 2007; FERGUNSON et al., 1999).

Desse modo, o objetivo desse estudo foi avaliar os efeitos de um PMTO e de sua cessação sobre a pressão arterial e frequência cardíaca de repouso em adolescentes, de acordo com o gênero.

\section{Métodos}

\section{Delineamento e sujeitos da pesquisa}

O estudo caracterizou-se como quaseexperimental com delineamento em séries de tempo (um momento com e outro sem tratamento) (THOMAS; NELSON, 2002). O programa foi divulgado na imprensa local durante o mês de fevereiro de 2010, convidando adolescentes com excesso de peso (sobrepeso e obesidade) e idade entre 10 e 18 anos para 0 ingresso nele. Foi realizada uma reunião com todos os interessados e seus responsáveis para maiores esclarecimentos das avaliações e do processo de intervenção.

Foram pré-determinados os seguintes critérios para inclusão no estudo: a) apresentarem disponibilidade para participar das intervenções nos horários e nos dias estipulados; b) apresentarem sobrepeso ou obesidade, segundo os pontos de corte para o IMC, idade e gênero, propostos por Cole et al. (2000); c) residirem em Maringá ou região metropolitana e; d) a concordância e assinatura do Termo de Consentimento Livre e Esclarecido (TCLE) do adolescente e de seu responsável a partir de documento aprovado pelo Comitê de Ética da UEM (parecer no 463/2009). Como critério de exclusão foi adotado fazer uso de qualquer medicamento que influenciasse no controle do peso corporal e da pressão arterial.

A intervenção foi conduzida no primeiro semestre de 2010 com 44 adolescentes, sendo 26 (59,09\%) do gênero feminino. Após a conclusão do período de 16 semanas de intervenção, os 22 (50\%) adolescentes concluintes foram convidados a retornarem para uma avaliação após um período de oito semanas sem intervenção multiprofissional, caracterizando - período de cessação das atividades do programa. Para esse período, os adolescentes receberam recomendações para a manutenção do estilo de vida saudável. No terceiro momento, retornaram apenas $19(43,18 \%)$ adolescentes que haviam iniciado o PMTO.

\section{Avaliações}

Após a assinatura do TCLE e preenchimento do cadastro (anamnese) utilizou-se um período de uma semana (semana pré-intervenção) para a aplicação dos seguintes testes:

\section{Avaliação Antropométrica e Composição Corporal}

Foram avaliadas massa corporal, estatura, circunferência da cintura (CC), abdominal (CA) e do quadril (CQ), IMC, massa gorda relativa (MGR) e massa magra (MM). Para verificar a massa corporal e a estatura dos adolescentes, foi utilizada uma balança da marca Welmy com 0,05 $\mathrm{Kg}$ de precisão e capacidade máxima de $300 \mathrm{Kg}$, e um estadiômetro acoplado a ela com precisão de $0,1 \mathrm{~cm}$ e que pode medir até dois metros de altura. Para a medida das circunferências, foi utilizada uma fita não-extensiva da marca Wiso de dois metros de cumprimento. O IMC foi calculado a partir da divisão do peso corporal pela altura ao quadrado (IMC = peso corporal/altura2). Todos os procedimentos foram padronizados seguindo as recomendações de Lohman, Roche e Martorell (1988).

A composição corporal dos adolescentes foi avaliada por meio de um aparelho de bioimpedância elétrica, multifrequencial, octapolar 
da marca InBody modelo 520. Os adolescentes foram aconselhados a seguirem as recomendações descritas por Heyward (2001): urinar cerca de 30 minutos antes da avaliação; abster-se do consumo de bebidas cafeinadas ao longo das últimas 48 horas; evitar esforços físicos vigorosos ao longo das últimas 24 horas; por fim, não utilizar diuréticos ao longo dos últimos sete dias. Para as meninas foi observado o período de ciclo menstrual para que este fator não interferisse nos resultados.

\section{Pressão arterial e frequência cardíaca de repouso}

Para essas avaliações foram usados um frequencímetro e esfigmomanômetro acoplados a um monitor não-invasivo configurável EMAI RX$300 \mathrm{~A}$. Os adolescentes permaneceram por 10 minutos sentados, em repouso, antes das medidas hemodinâmicas. A pressão arterial foi medida no braço direito, com o tamanho de manguito adequado para o adolescente sendo a medida feita na posição sentada. A pressão arterial sistólica foi definida como o aparecimento do primeiro som e a pressão arterial diastólica como o desaparecimento do som (LEITE et al., 2009). A pressão arterial foi classificada a partir de tabelas percentílicas específicas para adolescentes, levando-se em consideração seu gênero, idade e estatura. Os adolescentes foram categorizados em três grupos: normotensos, préhipertensos e hipertensos (NATIONAL HIGH BLOOD PRESSURE EDUCATION PROGRAM \begin{tabular}{llll}
\hline WORKING GROUP ON HIGH & BLOOD \\
\hline
\end{tabular} PRESSURE IN CHILDREN AND ADOLESCENTS, 2004).

As avaliações foram feitas em 3 momentos: a) avaliação pré-intervenção; b) avaliação pósintervenção (depois das 16 semanas de participação no PMTO); e c) avaliação pós período de cessação das atividades (8 semanas sem intervenção).

\section{Protocolo de Intervenção}

No período de intervenção, os adolescentes participaram de:

\section{Intervenção psicológica}

Realizada por uma psicóloga especialista em terapia cognitivo comportamental, com o auxílio de acadêmicas do curso de psicologia, através da realização de reuniões semanais em grupo com duração média de uma hora e finalidade de abordar os seguintes tópicos: a) estabelecimento de metas; b) auto-observação dos próprios comportamentos e consequências (imediatas, a médio prazo e a longo prazo); c) discriminação de sensações e análise de sentimentos; d) imagem corporal; e) ampliação do auto-conhecimento e análise dos eventos internos (pensamentos) e suas consequências; f) aspectos relacionados à auto-motivação e auto-controle; g) relacionamentos interpessoais (habilidades sociais).

\section{Intervenção nutricional}

Conduzida por duas nutricionistas experientes, por meio de reuniões semanais em grupo apenas com os adolescentes, com duração média de uma hora, com a finalidade de informar e orientar sobre aspectos nutricionais, tais como: pirâmide alimentar; densidade energética dos alimentos; importância dos macro e micronutrientes e sua relação com a saúde; composição nutricional dos alimentos; dentre outros.

\section{Intervenção dos profissionais da Educação Física}

Eram realizadas palestras semanais, a fim de fornecer informações e esclarecimentos sobre a prática de atividade física e seus benefícios (com duração média de uma hora), e atividade física orientada com frequência mínima de três vezes por semana (segundas, quartas e sextas-feiras) e duração média de uma hora por sessão. Foram priorizados os exercícios para grupos musculares localizados, por exemplo, abdominais, flexões de braço, agachamentos e exercícios com medicine ball (60-66\% FCmáx.) ( 20\% do tempo de intervenção), caminhada/corrida (54-59/75-84\% FCmáx.) ( 30\%) e 0 basquetebol (8289\%FCmáx.) ( 50\%).

\section{Intervenção médica}

Conduzida por um pediatra, através da realização de consultas individuais com os pais e adolescentes a fim de diagnosticar aspectos específicos do metabolismo de cada participante, como, por exemplo, patologias, além de orientálos sobre a importância de determinados hábitos e seus principais efeitos para o estado geral de saúde desses adolescentes.

\section{Análise Estatística}

Foi testada a normalidade dos dados, a partir do teste de Shapiro-Wilk. Os resultados foram tratados, estatisticamente, através da estatística descritiva (medidas de tendência central e dispersão) e inferencial (comparação entre momentos). Foi utilizado a Analysis of Variance 
(ANOVA) mista para medidas repetidas para a comparação entre os três momentos de acordo com o gênero e interação entre 0 fator independente (gênero) e o fator dependente (momentos), sendo a esfericidade avaliada a partir do teste de Mauchly. Para aqueles conjuntos de dados em que a esfericidade não foi assumida foi aplicado o ajuste de GreenhouseGeisser. Havendo diferença na ANOVA, foi conduzido o teste de comparações múltiplas com ajuste de Bonferroni. A significância foi préestabelecida em 5\%. Foram calculados os tamanhos de efeito (TE) ([média do pós-teste média do pré-teste]/desvio padrão do pré-teste) para determinar a magnitude dos efeitos da intervenção e do período de cessação. A magnitude do TE foi classificada de acordo com Cohen (1988) como: $\leq 0,20$ (trivial), entre $0,21 \mathrm{e}$ 0,50 (pequeno), entre 0,51 e 0,80 (moderado) e $>0,80$ (grande). $O$ estudo recebeu financiamento da Fundação Araucária.

\section{Resultados}

Dos 19 adolescentes que completaram o estudo, oito $(42,11 \%)$ eram do gênero feminino. A idade média da amostra foi de $13,00 \pm 1,70$ anos, sendo no gênero masculino de $12,91 \pm 1,58$ anos e no gênero feminino de $13,13 \pm 1,96$ anos. $\mathrm{Na}$ análise de normalidade dos dados de acordo com - gênero, todos os conjuntos de dados apresentaram normalidade.

No início da intervenção, $81,8 \%$ dos adolescentes do gênero masculino foram classificados como obesos e o restante como sobrepesados. Após o período de 16 semanas de intervenção, o número de obesos reduziu para $63,6 \%$, passando para $72,7 \%$ após as oito semanas de cessação do PMTO. Nas meninas, a prevalência de obesidade no início da intervenção foi de $87,5 \%$, mantendo-se após as 16 semanas de intervenção. Após o período de férias, esse número passou para $75 \%$. Nenhum adolescente foi classificado como eutrófico durante o estudo.

$\mathrm{Na}$ tabela 01 estão os resultados dos 19 adolescentes participantes desse estudo, divididos pelo gênero, antes da intervenção, após as 16 semanas e após o período de 2 meses de cessação, para as variáveis antropométricas e composição corporal.

Tabela 1. Efeitos do PMTO e de sua cessação sobre variáveis antropométricas e composição corporal antes da intervenção, após as 16 semanas e após o período de dois meses de cessação, nos adolescentes participantes do estudo $(n=19)$.

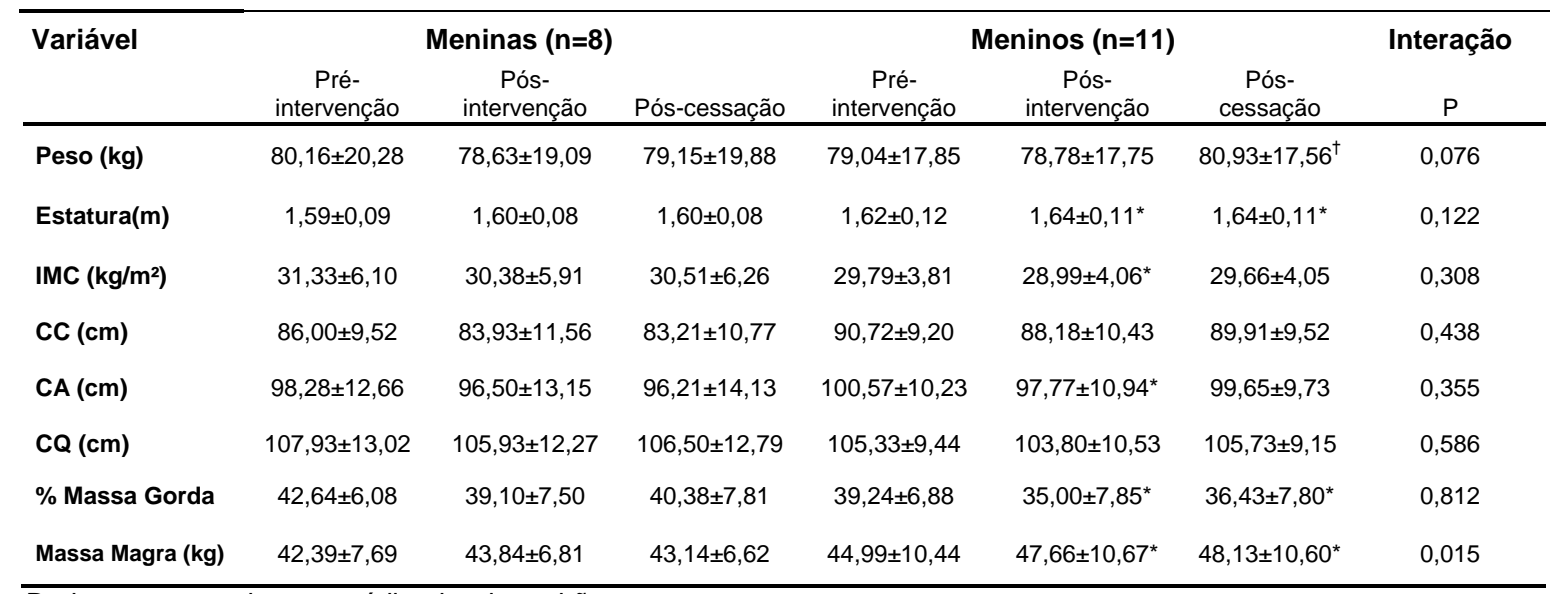

Dados apresentados em média desvio padrão.

CC - circunferência de cintura; CA - circunferência abdominal; $C Q$ - circunferência de quadril;

ANOVA mista para medidas repetidas; (*)Diferença estatisticamente significativa para o momento pré-intervenção no mesmo gênero; $(\dagger)$ Diferença estatisticamente significativa para o momento pós-intervenção no mesmo gênero.

No momento pré-intervenção, $27,3 \%$ dos meninos foram classificados como hipertensos, $18,2 \%$ como pré-hipertensos e $54,5 \%$ como normotensos. Após a intervenção, nenhum adolescente foi classificado com hipertensão arterial, $27,3 \%$ como pré-hipertensos e $72,7 \%$ como normotensos. Após o período de férias, o número de pré-hipertensos aumentou passando para $36,4 \%$, enquanto que 0 restante foi classificado como normotensos.

A tabela 02 apresenta os resultados referentes à pressão arterial e frequência cardíaca de repouso, de acordo com o gênero, antes da intervenção, após a intervenção e após o período de cessação das atividades. 
Tabela 2. Efeitos do PMTO e de sua cessação sobre a pressão arterial e frequência cardíaca de repouso antes da intervenção, após as 16 semanas e após o período de dois meses de cessação, nos adolescentes participantes do estudo $(n=19)$.

\begin{tabular}{|c|c|c|c|c|c|c|c|}
\hline \multirow[t]{2}{*}{ Variável } & \multicolumn{3}{|c|}{ Meninas $(n=8)$} & \multicolumn{3}{|c|}{ Meninos ( $n=11)$} & \multirow{2}{*}{$\begin{array}{c}\text { Gênero } x \text { momentos } \\
\text { P }\end{array}$} \\
\hline & $\begin{array}{c}\text { Pré- } \\
\text { intervenção }\end{array}$ & $\begin{array}{c}\text { Pós- } \\
\text { intervenção }\end{array}$ & $\begin{array}{c}\text { Pós- } \\
\text { cessação }\end{array}$ & $\begin{array}{c}\text { Pré- } \\
\text { intervenção }\end{array}$ & $\begin{array}{c}\text { Pós- } \\
\text { intervenção }\end{array}$ & Pós-cessação & \\
\hline PAS (mmHg) & $119,50 \pm 10,06$ & $114,50 \pm 11,15$ & $117,25 \pm 11,00$ & $121,91 \pm 13,88$ & $118,45 \pm 7,26$ & $113,09 \pm 9,19$ & 0,272 \\
\hline PAD (mmHg) & $69,38 \pm 7,21$ & $65,75 \pm 8,33$ & $67,63 \pm 12,27$ & $67,18 \pm 7,05$ & $63,82 \pm 4,38$ & $65,18 \pm 10,05$ & 0,993 \\
\hline FC (bpm) & $88,13 \pm 8,95$ & $78,00 \pm 6,99^{*}$ & $85,13 \pm 7,99$ & $77,55 \pm 11,01$ & $73,36 \pm 11,20$ & $74,73 \pm 12,08$ & 0,207 \\
\hline
\end{tabular}

Dados apresentados em média \pm desvio padrão

PAS - pressão arterial sistólica; PAD - pressão arterial diastólica; FC - frequência cardíaca.

ANOVA para medidas repetidas. ( $\left.{ }^{*}\right)$ Diferença estatisticamente significativa para o momento pós-intervenção no mesmo gênero.

Tabela 3. Tamanho de efeito das comparações entre os três momentos do estudo para o gênero feminino.

\begin{tabular}{lccc}
\hline \multirow{2}{*}{ Variável } & \multicolumn{3}{c}{ Comparações - meninas ( $\mathbf{n}=8)$} \\
\cline { 2 - 4 } & Pré-intervenção x pós-intervenção & Pré-intervenção x pós-cessação & Pós-intervenção x pós-cessação \\
\hline Peso (Kg) & $-0,08$ (trivial) & $-0,05$ (trivial) & 0,03 (trivial) \\
Altura (m) & 0,11 (trivial) & 0,11 (trivial) & 0 (trivial) \\
IMC (Kg/m²) & $-0,16$ (trivial) & $-0,13$ (trivial) & 0,02 (trivial) \\
CC (cm) & $-0,22$ (pequeno) & $-0,29$ (pequeno) & $-0,06$ (trivial) \\
CA (cm) & $-0,14$ (trivial) & $-0,16$ (trivial) & $-0,02$ (trivial) \\
CQ (cm) & $-0,15$ (trivial) & $-0,11$ (trivial) & 0,05 (trivial) \\
\% Massa Gorda & $-0,58$ (moderado) & $-0,37$ (pequeno) & 0,17 (trivial) \\
Massa Magra (kg) & 0,19 (trivial) & 0,10 (trivial) & $-0,10$ (trivial) \\
\hline PAS (mmHg) & $-0,50$ (pequeno) & $-0,22$ (pequeno) & 0,25 (pequeno) \\
PAD (mmHg) & $-0,51$ (moderado) & $-0,24$ (pequeno) & 0,23 (pequeno) \\
FC (bpm) & $-1,13$ (grande) & $-0,34$ (pequeno) & 1,02 (grande) \\
\hline
\end{tabular}

Tabela 4. Tamanho de efeito das comparações entre os três momentos do estudo para o gênero masculino.

\begin{tabular}{lccc}
\hline Variável & \multicolumn{3}{c}{ Comparações - meninos $(\mathbf{n}=\mathbf{1 1})$} \\
\hline Pesó-intervenção x pós-intervenção & $\begin{array}{c}\text { Pré-intervenção x pós- } \\
\text { cessação }\end{array}$ & $\begin{array}{c}\text { Pós-intervenção x pós- } \\
\text { cessação }\end{array}$ \\
\hline Altura (m) & $-0,01$ (trivial) & 0,11 (trivial) & 0,12 (trivial) \\
IMC (Kg/m²) & 0,17 (trivial) & 0,17 (trivial) & 0 (trivial) \\
CC (cm) & $-0,21$ (pequeno) & $-0,03$ (trivial) & 0,17 (trivial) \\
CA (cm) & $-0,28$ (pequeno) & $-0,09$ (trivial) & 0,17 (trivial) \\
CQ (cm) & $-0,27$ (pequeno) & $-0,09$ (trivial) & 0,17 (trivial) \\
\% Massa Gorda & $-0,16$ (trivial) & 0,04 (trivial) & 0,18 (trivial) \\
Massa Magra (kg) & $-0,62$ (moderado) & $-0,41$ (pequeno) & 0,18 (trivial) \\
\hline PAS (mmHg) & 0,26 (pequeno) & 0,30 (pequeno) & 0,04 (trivial) \\
PAD (mmHg) & $-0,25$ (pequeno) & $-0,64$ (moderado) & $-0,74$ (moderado) \\
FC (bpm) & $-0,48$ (pequeno) & $-0,28$ (pequeno) & 0,31 (pequeno) \\
\hline
\end{tabular}

As tabelas 03 e 04 descrevem os tamanhos de efeito e suas respectivas classificações para as comparações entre os três momentos no gênero feminino e masculino, respectivamente. 
No gênero feminino, no início da intervenção, $12,5 \%$ das meninas foram classificadas como hipertensas, $37,5 \%$ como pré-hipertensas e $50,0 \%$ como normotensas. Após a intervenção, não havia mais hipertensas no grupo e apenas $25 \%$ foram classificadas como pré-hipertensas. Com o período de férias, o número de préhipertensas aumentou para $62,5 \%$ enquanto que o restante permaneceu classificado como normotenso.

\section{Discussão}

O objetivo principal do presente estudo foi avaliar os efeitos de um PMTO e de sua cessação sobre a pressão arterial e frequência cardíaca de repouso em adolescentes, de acordo com o gênero.

Poucos são os estudos que fazem a análise dos efeitos de uma intervenção convencional a partir do gênero. Nosso trabalho indicou importantes melhoras após 16 semanas de intervenção multiprofissional sobre os parâmetros antropométricos e composição corporal de adolescentes do gênero masculino. Outros estudos demonstraram resultados semelhantes em relação à evolução do IMC após programas que fizeram uso de tratamentos convencionais de excesso de peso corporal em adolescentes, variando principalmente, o foco da intervenção (profissionais envolvidos) e o tempo de intervenção (4 semanas a 1 ano), porém a análise não foi dividida a partir do gênero (KASPRZAK; PILACZYŃSKA-SZCZEŚNIAK, 2010; SAVOYE et al., 2007; SABIA; SANTOS; RIBEIRO, 2004; CATTAI et al., 2008; COPPEN; RISSER; VASH, 2008; CHEN; ROBERTS; BARNARD, 2006).

Lofrano-Prado et al. (2009), após seis meses de intervenção multiprofissional, observaram nas meninas e nos meninos redução significativa do IMC e peso corporal, fato que se manteve após um ano do mesmo processo de intervenção. No entanto, há estudos que não encontraram a mesma diferença, principalmente para o peso corporal, porém nesses casos a análise não foi feita a partir do gênero (SABIA; SANTOS; RIBEIRO, 2004; CATTAl et al., 2008). Isso pode estar associado ao fato de que, com a prática de exercícios físicos, há diminuição da massa de gordura corporal, no entanto, observa-se aumento da massa muscular esquelética, o que reflete em uma pequena alteração no peso corporal. Além disso, os adolescentes estão em fase de crescimento o que pode aumentar a sua massa corporal.

Especificamente, em relação às medidas de circunferência de cintura, abdômen e quadril foi observada melhora estatisticamente significativa apenas para a circunferência de abdômen nos meninos (tamanho de efeito pequeno). Um estudo utilizando o mesmo modelo de tratamento, durante o mesmo período, também encontrou melhora significativa para a circunferência abdominal, assim como para a circunferência de cintura de adolescentes (CATTAl et al., 2008).

Em nosso estudo, após 16 semanas de intervenção, houve redução da massa gorda relativa (tamanho de efeito moderado) e aumento da massa magra (tamanho de efeito pequeno) apenas para os meninos com interação entre gênero e momentos de avaliação para esta última variável, demonstrando relação entre os efeitos obtidos durante a intervenção com o gênero do adolescente. $O$ estudo de Lofrano-Prado et al. (2009) não encontrou alterações para a massa livre de gordura tanto nas meninas quanto nos meninos em seis meses de acompanhamento. Nesse mesmo estudo, para a massa gorda, apenas os meninos apresentaram redução após esse período, semelhante ao observado em nosso estudo.

A análise do período pós-cessação das atividades do PMTO mostrou aumento significativo do peso corporal dos meninos. Gillis, McDowell e Bar-or (2005) observaram, em uma análise feita com os dois gêneros em conjunto, que $66 \%$ dos adolescentes ganharam peso no período de férias. Em contrapartida, Togashi et al. (2002) acompanharam adolescentes por um período médio de um ano, oferecendo intervenção com foco na mudança do hábito alimentar e de atividade física e depois os avaliaram quando adultos (11,8 $\pm 3,7$ anos após). Do grupo que iniciou a intervenção com obesidade severa $(n=128), 34$ tornaram-se eutróficos na fase adulta, 47 foram classificados, quando adultos, como sobrepesados e os outros 47, permaneceram obesos.

$\mathrm{Na}$ análise dos dados de composição corporal dos adolescentes após o período de cessação das atividades de nosso programa, observamos a manutenção das melhoras obtidas no gênero masculino enquanto que para as meninas os resultados do baseline foram mantidos. Resultados contrários foram obtidos por $\underline{\text { Carrell et }}$ 
al. (2007) que observaram que três meses de cessação de atividades físicas foram suficientes para aumentarem a massa gorda dos adolescentes envolvidos. Para os autores, se não estiverem sempre engajados, os jovens não conseguem manter os benefícios que obtiveram na intervenção. O estudo de Fergunson et al. (1999) teve resultados semelhantes. O grupo que fez exercícios físicos apenas por quatro meses diminuiu seu percentual de gordura, porém após quatro meses de cessação, os resultados foram perdidos. O grupo que começou os primeiros quatro meses sem exercícios físicos, não apresentou alteração no percentual de gordura, no entanto, após serem submetidos à intervenção, houve redução da gordura corporal.

Mudanças em parâmetros antropométricos e composição corporal em adolescentes podem ser influenciadas não só pelo tratamento em questão, mas também por outros fatores fisiológicos. 0 desenvolvimento sexual que ocorre durante a adolescência promove alterações endócrinometabólicas que influenciam na distribuição de gordura corporal tanto nos meninos como nas meninas. $O$ gênero feminino tende a apresentar maior depósito de gordura em regiões periféricas, como, os seios, quadril e nádegas. Os rapazes, por outro lado, apresentam, a partir desse período, maior centralização da gordura corporal e aumento dos estoques de gordura subcutânea e visceral na região abdominal. Assim, as mudanças observadas no presente estudo podem estar associadas a esses fatores (CARANTI et al., 2007; MALINA; BOUCHARD, 2002).

Os valores médios da pressão arterial dos adolescentes apresentaram-se dentro da normalidade no momento pré-intervenção, considerando 0 valor estabelecido pelo International Diabetes Federation (2007) (130/85 $\mathrm{mmHg}$ ), para diagnóstico de Síndrome Metabólica, em crianças e adolescentes e os valores específicos para idade, gênero e estatura do National High Blood Pressure Education Program Working Group on High Blood Pressure in Children and Adolescents (2004). Além disso, apenas quatro adolescentes $(21,1 \%)$ foram classificados como hipertensos no início da intervenção, sendo um do gênero feminino ( $12,5 \%$ do total de oito meninas) e três do gênero masculino (27,3\% do total de 11 meninos).

No presente estudo, não foram encontradas diferenças significativas nos valores de pressão arterial sistólica e diastólica após 16 semanas de intervenção multiprofissional, apresentando um tamanho de efeito pequeno para ambos os gêneros na maioria das comparações. Houve diminuição significativa da frequência cardíaca de repouso no gênero feminino.

Apesar de nossos dados corroborarem com alguns trabalhos encontrados na literatura em relação aos resultados da intervenção sobre o perfil hemodinâmico de adolescentes obesos, há trabalhos que mostram resultados contrários, ou seja, melhoras significativas nas variáveis, pressão arterial sistólica e diastólica por um período de duas semanas de tratamento intensivo realizado em ambiente diferente do usual e com rotina também especificamente organizada para os fins do programa ( $\underline{\mathrm{CHEN}}$; ROBERTS; BARNARD, 2006) e por 10 semanas de intervenção, tanto em meninos quanto em meninas (COPPEN; RISSER; VASH, 2008).

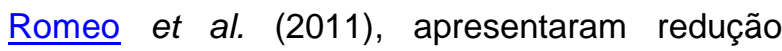
significativa somente na pressão arterial diastólica após 13 meses de um programa baseado em dieta de restrição calórica (10-40\%), aumento da atividade física (pelo menos $60 \mathrm{~min} /$ dia 5 dias por semana), terapia psicológica e educação nutricional, sendo a análise feita sem estratificação por gênero.

Leite et al. (2009) analisaram, em seu estudo, os efeitos de uma intervenção multiprofissional, com exercício físico e orientação alimentar, em adolescentes obesos com e sem Síndrome Metabólica (SM). Nesse estudo, no grupo com SM, foi observada diminuição significativa da pressão arterial sistólica após 12 semanas de intervenção, enquanto que para a pressão arterial diastólica não houve nenhuma diferença. No grupo sem SM, não houve diferença entre os momentos pré e pós-intervenção para as duas variáveis, semelhante ao observado ao nosso estudo. Shrewsbury et al. (2011) realizaram dois meses de intervenção com equipe multiprofissional e também não observaram alterações sobre a pressão arterial em adolescentes sobrepesados.

Em adolescentes com a mesma característica, porém sendo a análise feita por gênero, Caranti et al. (2007) verificaram os efeitos de uma intervenção multiprofissional, oferecida por educadores físicos, nutricionistas, médicos e psicólogos em adolescentes obesos. Nesse trabalho, um período de intervenção de seis 
meses, não causou mudanças significativas na pressão arterial sistólica e diastólica no gênero feminino, assim como um ano de intervenção para o mesmo gênero. No entanto, para o gênero masculino foi observada melhora significativa da pressão arterial sistólica na comparação entre o baseline e o momento pós- 6 meses de intervenção e entre o baseline e o momento pósum ano de intervenção. Contraditoriamente, em um estudo feito no ambiente escolar com 2.494 crianças obesas de 6 a 13 anos, Hollar et al. (2010) encontraram diminuição significativa na pressão arterial sistólica e diastólica entre meninas dos grupos intervenção em comparação ao controle após 1 ano de intervenção multiprofissional. Não houve diferenças significativas entre meninos.

Há uma diferença importante entre alguns trabalhos presentes na literatura que pode explicar nossos resultados referentes à pressão arterial: o valor dessa variável no baseline. No estudo de Chen, Roberts e Barnard (2006), por exemplo, o valor médio inicial da pressão arterial foi de $130 / 74,3 \mathrm{mmHg}$. No trabalho de Leite et al. (2009), o valor médio da pressão arterial, no início para o grupo sem síndrome metabólica, foi de $99,73 / 64,26 \mathrm{mmHg}$. Em nosso trabalho, a maioria dos adolescentes também iniciou o tratamento com valores dentro da normalidade. Consequentemente, é possível que, por esse motivo, não tenham sido identificadas diferenças na pressão arterial desses adolescentes com excesso de peso, após terem sido submetidos a nossa intervenção. Mesmo assim, quando analisada a prevalência de hipertensão arterial na amostra, os valores reduziram para os dois gêneros, sendo que após a intervenção nenhum adolescente foi classificado com hipertensão arterial. Entretanto, após o período de cessação das atividades do PMTO, o número de adolescentes com pré-hipertensão arterial aumentou em comparação ao momento pósintervenção.

Apesar dos mecanismos que induzem à redução da pressão arterial em sujeitos que reduziram a gordura corporal permanecerem desconhecidos, segundo Mertens e Van Gaal (2000) esses resultados podem estar ligados à redução do volume de fluído extracelular, redução do débito cardíaco, redução da atividade do sistema nervoso simpático, aumento à sensibilidade à insulina e normalização do sistema renina-angiotensina-aldosterona.
Em relação à frequência cardíaca de repouso, verificamos redução significativa após 16 semanas para o gênero feminino. Fergunson et al. (1999) realizaram um estudo de oito meses, com dois grupos de jovens obesos, com idade entre sete e 11 anos: o primeiro recebeu intervenção com exercícios físicos, apenas por quatro meses, ficando os quatro meses, posteriores, sem intervenção. $O$ segundo grupo fez o contrário. A avaliação da frequência cardíaca submáxima indicou que, no período com intervenção, os participantes do estudo apresentaram melhores valores desse parâmetro, comparados ao momento em que os mesmos não participaram da intervenção e seguiram suas rotinas, demonstrando possivelmente um melhor condicionamento aeróbio (ALMEIDA; ARAÚJO, 2003). Em relação à frequência cardíaca no repouso, valores mais baixos podem estar associados a um quadro de saúde mais favorável. Esses valores mais baixos podem refletir um maior controle autonômico refletido por uma maior atividade parassimpática, o que estaria ligado a um menor risco para doenças cardiovasculares (GREENLAND et al., 1999; SPALDING et al., 2000).

Apesar das contribuições para a área por envolver na análise um período de cessação das atividades e análise estratificada por gênero, nosso estudo apresenta algumas limitações. Nossa amostra foi composta apenas por 19 sujeitos, sendo que tivemos uma grande perda amostral, durante o processo de intervenção $(56,82 \%)$, por motivos diversos, tais como: incompatibilidade de horários, motivação unilateral (apenas do responsável ou do adolescente), dificuldades com 0 transporte e realização de procedimentos cirúrgicos sem relação com o quadro de obesidade (cirurgia dentária e cirurgia relacionada à fratura de osso do quadril devido a acidente automobilístico). Além disso, não foi possível avaliar o nível de atividade física e o consumo alimentar, variáveis importantes para avaliação da mudança do comportamento alimentar e de atividade física de um indivíduo, além de influenciarem nas variáveis hemodinâmicas avaliadas.

Em resumo, os resultados desse estudo demonstraram que 16 semanas de intervenção promovem melhorias sobre os parâmetros antropométricos e composição corporal de adolescentes com excesso de peso do gênero masculino e sobre a frequência cardíaca de 
repouso do gênero feminino. Após o período de cessação de oito semanas, nenhuma mudança foi verificada, com exceção do aumento do peso corporal no gênero masculino, porém sem alteração das melhoras obtidas sobre a composição corporal dos mesmos.

Sugerimos para futuros trabalhos intervenções com uma duração superior a 16 semanas, fazendo uso da abordagem cognitivo comportamental e que tenham um foco maior na avaliação de períodos em que os adolescentes ficam sem intervenção. Além disso, estudos com uma amostra maior e mais bem controlados podem ser feitos para esclarecer as diferenças entre os gêneros que ainda são contraditórias. Parâmetros bioquímicos, como a glicemia em jejum, o colesterol total e fracionado, triglicerídeos e insulinemia e parâmetros do estilo de vida (alimentação e prática habitual de atividade física) devem ser avaliados, para verificar os efeitos desse modelo de intervenção e de sua cessação em outros parâmetros relacionados à síndrome metabólica.

\section{Referências}

ALMEIDA, M. B., ARAÚJO, C. G. S. Efeitos do treinamento aeróbico sobre a frequência cardíaca. Revista Brasileira de Medicina do Esporte, Rio de Janeiro, v. 9, n. 2, p. 10-12, 2003. Disponível em:

http://www.scielo.br/pdf/rbme/v9n2/v9n2a06.pdf. Acesso em 23 de abril de 2010.

BAO, W., THREEFOOT, S. A., SRINIVASAN, S. R., BERENSON, G. S. Essential hypertension predicted by tracking of elevated blood pressure from childhood to adulthood: the Bogalusa Heart Study. American Journal of Hypertension, New York, v. 8, n. 7, p. 657-65, 1995. Disponível em: http://www.nature.com/ajh/journal/v8/n7/full/ajh199 592a.html. Acesso em 23 de abril de 2010.

CAMPOS, W., STABELINI NETO, A., BOZZA, R., ULBRICH, A. Z., BERTIN, R. L.,

MASCARENHAS, L. P., SILVA, S. G., SASAKI, J. E. Physical activity, lipid consumption and risk factors for atherosclerosis in adolescents.

Arquivos Brasileiros de Cardiologia, São Paulo, v. 94, n. 5, p. 601-7, 2010. Disponível em: http://www.scielo.br/pdf/abc/v94n5/es aop02810.p df. Acesso em 17 de maio de 2010.

CARANTI, D. A.; MELLO, M. T.; PRADO, W. L., TOCK, L., SIQUEIRA, K. O., DE PIANO, A., LOFRANO, M. C., CRISTOFALO, D.M., LEDERMAN, H., TUFIK, S., DẦMASO, A.R. Short- and long-term beneficial effects of a multidisciplinary therapy for the control of metabolic syndrome in obese adolescents.

Metabolism Clinical and Experimental, Naples, v. 56, p. 1293-1300, 2007. Disponível em: http://www.metabolismjournal.com/article/S00260495(07)00185-0/pdf. Acesso em: 20 de novembro de 2009.

CARELL, A. L., CLARK, R.R, PETERSON, S., EICKHOFF, J., ALLEN, D.B. School-based fitness changes are lost during summer vacation.

Archives Pediatric Adolescent Medicine, Seattle, v. 161, p. 561-564, 2007. Disponível em: http://archpedi.ama-

assn.org/cgi/reprint/161/6/561. Acesso em 20 de novembro de 2009.

CATTAI, G. B. P., ROCHA, F. A., HINTZE L. J., PAGAN, B. G. M., NARDO-JUNIOR, N. Programa de Tratamento Multiprofissional da Obesidade: os desafios da prática. Ciência, Cuidado e Saúde, Maringá, v. 7(Suplem. 1), p. 121-6, 2008.

Disponível em:

http://www.mendeley.com/research/programatratamento-multiprofissional-da-obesidade-osdesafios-da-prtica/\#page-1. Acesso em $30 \mathrm{de}$ setembro de 2009.

CDC. Participation in High School Physical Education - United States 1991-2003. MMWR CDC Suveill Summ, Atlanta, v. 53, 844-847, 2004. Disponível em:

http://www.cdc.gov/mmwr/preview/mmwrhtml/mm 5336a5.htm. Acesso em 20 de novembro de 2009.

CHEN, A. K., ROBERTS, C. K., BARNARD, R. J. Effect of a short-term diet and exercise intervention on metabolic syndrome in overweight children. Metabolism Clinical and Experimental, Naples, v. 55, p.871-8, 2006. Disponível em: http://download.journals.elsevierhealth.com/pdfs/jo urnals/0026-0495/PIIS0026049506000758.pdf. Acesso em 20 de novembro de 2009.

COHEN, J. Statistical power analysis for the behavioral sciences. Hillsdale, Lawrence Erlbaum, 1988.

COLE, T. J., BELLIZZI, M. C., FLEGAL, K. M., DIETZ, W. H. Establishing a standard definition for child overweight and obesity worldwide: international survey. British Medical Journal, Londres, v. 320, p. 1240-1243, 2000. Disponível em:

http://www.ncbi.nlm.nih.gov/pmc/articles/PMC273 65/?tool=pubmed. Acesso em 08 de fevereiro de 2008.

COPPEN, A. M.; RISSER, J. A.; VASH, P. D. Metabolic Syndrome Resolution in Children and Adolescents After 10 Weeks of Weight Loss. Journal of the CardioMetabolic 
Syndrome, Malden, p. 205-10, 2008. Disponível em:

http://onlinelibrary.wiley.com/doi/10.1111/j.15594572.2008.00016.x/pdf. Acesso em 20 de novembro de 2009.

FERGUSON, M. A., GUTIN, B., LE, N. A., KARP, W., LITAKER, M., HUMPHRIES, M., OKUYAMA, T., RIGGS, S., OWENS, S. Effects of exercise training and its cessation on components of the insulin resistance syndrome in obese children. International Journal of Obesity and Related Metabolic Disorders, Virginia, v. 23, n. 8, p. 88995, 1999. Disponível em:

http://www.nature.com/ijo/journal/v23/n8/abs/0800 968a.html. Acesso em 20 de novembro de 2009.

FERREIRA, J. S.; AYDOS, R. D. Prevalência de hipertensão arterial em crianças e adolescentes obesos. Ciência \& Saúde Coletiva, Rio de Janeiro, v. 15, n. 1, p. 97-104, 2010. Disponível em:

http://www.scielo.br/pdf/csc/v15n1/a15v15n1.pdf. Acesso em 23 de abril de 2010.

GILLIS, L., MCDOWELL, M., BAR-OR, O. Relationship between summer vacation weight gain and lack of success in a pediatric weight control program. Eating Behaviors, Charleston, v. 6, p. 137-43, 2005. Disponível em: http://www.sciencedirect.com/science/article/pii/S1 471015304000558 . Acesso em 20 de novembro de 2009.

GREENLAND, P., DAVIGLUS, M. L., DYER, A. R., LIU, K., HUANG, C. F., GOLDBERGER, J. J., STAMLER, J. Resting heart rate is a risk factor for cardiovascular and noncardiovascular mortality: the Chicago Heart Association Detection Project in Industry. American Journal of Epidemiology. Oxford, v. 149, p. 853-62, 1999. Disponível em: http://aje.oxfordjournals.org/content/149/9/853.full. pdf. Acesso em 23 de abril de 2010.

HEYWARD, V. H. ASEP methods recommendation: body composition assessment. Journal of Exercise Physiology, Duluth, v. 4, p. 1-12, 2001. Disponível em: http://faculty.css.edu/tboone2/asep/HeywardFinal. pdf. Acesso em 14 de julho de 2009.

HOLLAR, D., MESSIAH, S. E., LOPEZ-MITNIK, G., HOLLAR, T. L., ALMON, M., AGATSTON, A. S. Healthier options for public schoolchildren program improves weight and blood pressure in 6to 13-year-olds. Journal of the American

Dietetic Association, Oxford, v. 110, n. 2, p. 2617, 2010. Disponível em:

http://www.adajournal.org/article/S00028223(09)01805-7/pdf. Acesso em 23 de abril de 2010.
IBGE - Instituto Brasileiro de Geografia e Estatística. Pesquisa de Orçamentos Familiares 2008-2009: desnutrição cai e peso das crianças brasileiras ultrapassa padrão internacional. 2010. Disponível

em: http://www.ibge.gov.br/home/presidencia/notic ias/noticia visualiza.php?id noticia=1699\&id pagi na=1 Acesso em 23 de fevereiro de 2011.

IDF International Diabetes Federation. The IDF Consensus Definition of the Metabolic Syndrome in Children and

Adolescents. Brussels, Belgium, 2007, p. 2-19. Disponível em:

http://www.idf.org/webdata/docs/Mets definition c hildren.pdf. Acesso em 20 de novembro de 2009.

KASPRZAK, Z., PILACZYŃSKA-SZCZEŚNIAK, L. Effect of diet and physical activity on physiological and biochemical parameters of obese adolescents. Acta Science Polish Technology Alimentary, Olsztyn, v. 9, n. 1, p. 95-104, 2010. Disponível em: http://www.food.actapol.net/pub/8 1 2010.pdf. Acesso em 20 de novembro de 2009.

LEITE, N.; MILANO, G. E.; CIESLAK, F. et al. Effects of physical exercise and nutritional guidance on metabolic syndrome in obese adolescents. Revista Brasileira de Fisioterapia, São Carlos, v. 13, n. 1, p. 73-81, 2009. Disponível em:

http://www.scielo.br/pdf/rbfis/v13n1/007 09.pdf. Acesso em 20 de novembro de 2009.

LOFRANO-PRADO, M. C., ANTUNES, H. K. M., PRADO, W. L., DE PIANO, A., CARANTI, D. A., TOCK, L., CARNIER, J., TUFIK, S., DE MELLO, M. T., DÂMASO, A. R. Quality of life in Brazilian obese adolescents: effects of a longterm multidisciplinary lifestyle therapy. Health and Quality of Life Outcomes, Londres, v. 7, p. 61-8, 2009. Disponível em:

http://www.ncbi.nlm.nih.gov/pmc/articles/PMC271 3214/pdf/1477-7525-7-61.pdf. Acesso em 23 de abril de 2010.

LOHMAN, T. G., ROCHE, A. F., MARTORELL, R. Anthropometric standardization reference manual. Champaign: Human Kinetics, 1988.

MALINA, R. M., BOUCHARD, C. Atividade física do atleta jovem: do crescimento à maturação. $1^{\text {a }}$ Ed. Roca: São Paulo, 2002.

MERTENS, I. L., VAN GAAL, L. F. Overweight, obesity and blood pressure: the effects of modest weight reduction. Obesity Research, New York, v. 8, p. 270-8, 2000. Disponível em: http://www.ncbi.nlm.nih.gov/pubmed/10832771. Acesso em 23 de abril de 2010. 
NATIONAL HIGH BLOOD PRESSURE EDUCATION PROGRAM WORKING GROUP ON HIGH BLOOD PRESSURE IN CHILDREN AND ADOLESCENTS. The fourth report on the diagnosis, evaluation, and treatment of high blood pressure in children and adolescents. Pediatrics, Houston, v. 114, n. 2, suppl. 4, p. 555-76, 2004. Disponível em:

http://pediatrics.aappublications.org/content/114/S upplement 2/555.full.pdf+html. Acesso em: 23 de abril de 2010.

POLDERMAN, J., GURGEL, R. Q., BARRETOFILHO, J. A., ROELOFS, R., RAMOS, R. E., DE MUNTER, J. S., WENDTE, J. F., AGYEMANG, C. Blood pressure and BMI in adolescents in Aracaju, Brazil. Public Health Nutrition, Cambridge, v. 14, n. 6, p. 1064-70, 2011. Disponível em:

http://journals.cambridge.org/action/displayAbstrac t?fromPage $=$ online\&aid=8269931. Acesso em: 23 de abril de 2010.

QUEIROZ, V. M., MOREIRA, P. V., VASCONCELOS, T. H., VIANNA, R. P. T. Prevalence and anthropometric predictors of high blood pressure in school children from João Pessoa - PB, Brazil. Arquivos Brasileiros de Cardiologia, São Paulo, v. 95, n. 5, p. 629-34, 2010. Disponível em:

http://www.scielo.br/pdf/abc/v95n5/en v95n5a11.p df. Acesso em 23 de fevereiro de 2011.

ROLLAND-CACHERA, M. F. THIBAULT, H., SOUBERBIELLE, J. C., SOULIÉ, D., CARBONEL, P., DEHEEGER, M., ROINSOL, D., LONGUEVILLE, E., BELLISLE, F., SEROG, P. Massive obesity in adolescents: dietary interventions and behaviours associated with weight regain at $2 y$ follow-up. International Journal of Obesity, Londres, v. 28, p. 514-9, 2004. Disponível em: http://www.nature.com/ijo/journal/v28/n4/pdf/0802 605a.pdf. Acesso em 20 de novembro de 2009.

ROMEO, J., MARTINEZ-GOMEZ, D., DIAZ, L. E., GÓMEZ-MARTINEZ, S., MARTI, A., MARTINMATILLAS, M., PUERTOLLANO, M. A., VEIGA, O. L., MARTINEZ, J. A., WÄRNBERG, J., ZAPATERA, B., GARAGORRI, J. M., MORANDÉ, G., CAMPOY, C., MORENO, L. A., MARCOS, A., EVASYON STUDY GROUP. Changes in cardiometabolic risk factors, appetite-controlling hormones and cytokines after a treatment program in overweight adolescents: preliminary findings from the EVASYON study. Pediatric Diabetes, São Francisco, v. 12, n. 4Pt2, p. 37280, 2011. Disponível em:

http://onlinelibrary.wiley.com/doi/10.1111/i.13995448.2010.00753.x/pdf. Acesso em 20 de novembro de 2009.

SABIA, R. V., SANTOS, J. E., RIBEIRO, R. P. P. Efeito da atividade física associada à orientação alimentar em adolescentes obesos: comparação entre o exercício aeróbio e anaeróbio. Revista Brasileira de Medicina do Esporte, Rio de Janeiro, v. 10, n. 5, p. 349-55, 2004. Disponível em:

http://www.scielo.br/pdf/rbme/v10n5/v10n5a02.pdf . Acesso em 04 de julho de 2008.

SAVOYE, M., SHAW, M., DZIURA, J., TAMBORLANE, W. V., ROSE, P., GUANDALINI, C., GOLDBERG-GELL, R., BURGERT, T. S., CALI, A. M., WEISS, R., CAPRIO, S. Effects of a Weight Management Program on Body Composition and Metabolic Parameters in Overweight Children: A Randomized Controlled Trial. Journal of American Medical Association, Chicago, v. 297, n. 24, p. 2697-704, 2007. Disponível em: http://jama.ama-

assn.org/content/297/24/2697.full.pdf+html. Acesso em 20 de novembro de 2009.

SHREWSBURY, V. A., NGUYEN, B., O'CONNOR, J., STEINBECK, K. S., LEE, A., HILL, A. J., SHAH, S., KOHN, M. R., TORVALDSEN, S., BAUR, L. A. Short-term outcomes of community-based adolescent weight management: The Loozit® Study. BMC Pediatric, Londres, v. 11, n. 13, 2011. Disponível em: http://www.ncbi.nlm.nih.gov/pmc/articles/PMC305 0717/pdf/1471-2431-11-13.pdf. Acesso em 20 de novembro de 2009.

SOUZA, M. G., RIVERA, I. R., SILVA, M. A., CARVALHO, A. C. Relationship of obesity with high blood pressure in children and adolescents. Arquivos Brasileiros de Cardiologia, São Paulo, v. 94, n. 6, p. 714-9, 2010. Disponível em: http://www.scielo.br/pdf/abc/v94n6/en aop03710.p df. Acesso em 04 de julho de 2011.

SPALDING, T. W., JEFFERS, L. S., PORGES, S. W., HATFIELD, B. D. Vagal and cardic reactivity to psychological stressors in trained and untrained men. Medicine \& Science in Sports \& Exercise, Indianapolis, v. 32, p. 581-91, 2000. Disponível em: http://journals.lww.com/acsmmsse/pages/articleviewer.aspx?year=2000\&issue $=03000 \&$ article $=00006 \&$ type $=$ abstract. Acesso em: 23 de abril de 2010.

THOMAS, J. R.; NELSON, J. K. Métodos de Pesquisa em Atividade Física/ trad. Ricado Petersen, et al. - $3^{\mathrm{a}}$ ed. - Porto Alegre: Artmed, 2002.

TOGASHI, K., MASUDA, H., RANKINEN, T., TANAKA, S., BOUCHARD, C., KAMIYA, H. A 12year follow-up study of treated obese children in Japan. International Journal of Obesity, Londres, v. 26, p. 770-7, 2002. Disponível em: http://www.nature.com/ijo/journal/v26/n6/pdf/0801 992a.pdf. Acesso em 20 de novembro de 2009. 
WHELTON, S. P., CHIN, A., XIN, X., HE, J. Effect of aerobic exercise on blood pressure: a metaanalysis of randomized, controlled trials. Annals of Internal Medicine, Philadelphia, v. 136, n. 7, p. 493-505, 2002. Disponível em:

http://www.annals.org/content/136/7/493.full.pdf+h tml. Acesso em 23 de abril de 2010.

WHO European Ministerial Conference on Counteracting Obesity: Diet and Physical

Activity for Health. Istambul, Turkey, 2006.

Disponível em:

http://www.euro.who.int/ data/assets/pdf file/000 6/96459/E90143.pdf. Acesso em 26 de agosto de 2009.

Apoio financeiro: Fundação Araucária

Agradecimentos: Agradecemos aos membros do Núcleo de Estudos Multiprofissional da Obesidade que contribuíram com as coletas de dados e realização da intervenção em questão. Agradecemos também à Fundação Araucária.

\section{Endereço:}

Danilo Fernandes da Silva

UEM - Depto. de Educação Física

Av. Colombo, 5.790 Bairro: Jardim Universitário Maringá, PR, Brasil

87020-900

Telefone: (44) 3011- 4315

e-mail: danilofernandesdasilva@hotmail.com

Recebido em: 24 de novembro de 2011.

Aceito em: 21 de março de 2013.

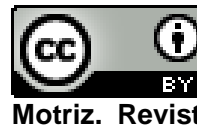

Motriz. Revista de Educação Física. UNESP, Rio Claro, SP, Brasil - elSSN: 1980-6574 - está licenciada sob Creative Commons - Atribuição 3.0 